\title{
Certain $\boldsymbol{m}$-Convexity Inequalities Related to Fractional Integrals with Exponential Kernels
}

\author{
Hao Wang*, Zhijuan Wu \\ Department of Mathematics, College of Science, Hunan City University, Yiyang, China \\ Email: *haowangctgu@163.com, 444067457@qq.com
}

How to cite this paper: Wang, $\mathrm{H}$. and $\mathrm{Wu}$, Z.J. (2021) Certain $m$-Convexity Inequalities Related to Fractional Integrals with Exponential Kernels. Open Access Library Journal, 8: e7388.

https://doi.org/10.4236/oalib.1107388

Received: April 5, 2021

Accepted: May 17, 2021

Published: May 20, 2021

Copyright $\odot 2021$ by author(s) and Open Access Library Inc.

This work is licensed under the Creative Commons Attribution International License (CC BY 4.0).

http://creativecommons.org/licenses/by/4.0/

(c) (i) Open Access

\begin{abstract}
In this paper, we establish some mid-point type and trapezoid type inequalities via a new class of fractional integral operators which is introduced by Ahmad et al. We derive a new fractional-type integral identity to obtain Dragomir-Agarwal inequality for $m$-convex mappings. Moreover, some inequalities of Hermite-Hadamard type for $m$-convex mappings are given related to fractional integrals with exponential kernels. The results presented provide extensions of those given in earlier works.
\end{abstract}

\section{Subject Areas}

Mathematical Analysis, Numerical Mathematics

\section{Keywords}

Hermite-Hadamard Type Inequality, Fractional Integrals, $m$-Convex Mappings

\section{Introduction}

Recently, Ahmad et al. [1] presented a new fractional integral operator, which is named fractional integrals with exponential kernels, as follows.

Definition 1.1. Let $\varphi \in L^{1}([a, b])$. The fractional integrals $I_{\gamma_{1}^{+}}^{\mu} \varphi$ and $I_{\gamma_{2}^{-}}^{\mu} \varphi$ of order $\mu \in(0,1)$ are defined respectively by

$$
I_{\gamma_{1}^{+}}^{\mu} \varphi(x)=\frac{1}{\mu} \int_{\gamma_{1}}^{x} \mathrm{e}^{-\frac{1-\mu}{\mu}(x-\tau)} \varphi(\tau) \mathrm{d} \tau, \quad x \geq \gamma_{1},
$$

and

$$
I_{\gamma_{2}}^{\mu} \varphi(x)=\frac{1}{\mu} \int_{x}^{\gamma_{2}} \mathrm{e}^{-\frac{1-\mu}{\mu}(\tau-x)} \varphi(\tau) \mathrm{d} \tau, \quad x \leq \gamma_{2}
$$

${ }^{\star}$ Corresponding author. 
Note that

$$
\lim _{\mu \rightarrow 1} I_{\gamma_{1}^{+}}^{\mu} \varphi(x)=\int_{\gamma_{1}}^{x} \varphi(\tau) \mathrm{d} \tau \text { and } \lim _{\mu \rightarrow 1} I_{\gamma_{2}^{-}}^{\mu} \varphi(x)=\int_{x}^{\gamma_{2}} \varphi(\tau) \mathrm{d} \tau .
$$

In [1], the authors obtained new versions of Hermite-Hadanard inequality based on this fractional integral operators as follows.

Theorem 1.1. Let $\varphi:\left[\gamma_{1}, \gamma_{2}\right] \rightarrow \mathbb{R}$ be a non-negative convex mapping and $0 \leq \gamma_{1}<\gamma_{2}<\infty$. If $\varphi \in L^{1}\left(\left[\gamma_{1}, \gamma_{2}\right]\right)$, then the following double inequalities hold:

$$
\varphi\left(\frac{\gamma_{1}+\gamma_{2}}{2}\right) \leq \frac{1-\mu}{2\left(1-\mathrm{e}^{-\rho}\right)}\left[I_{\gamma_{1}^{+}}^{\mu} \varphi\left(\gamma_{2}\right)+I_{\gamma_{2}^{-}}^{\mu} \varphi\left(\gamma_{1}\right)\right] \leq \frac{\varphi\left(\gamma_{1}\right)+\varphi\left(\gamma_{2}\right)}{2}
$$

where $\rho=\frac{1-\mu}{\mu}\left(\gamma_{2}-\gamma_{1}\right)$.

Taking $\mu \rightarrow 1$ i.e. $\rho=\frac{1-\mu}{\mu}\left(\gamma_{2}-\gamma_{1}\right) \rightarrow 0$ in Theorem 1.1, we can recapture classical Hermite-Hadamard inequality for a convex function $\varphi$ on $\left[\gamma_{1}, \gamma_{2}\right]$ :

$$
\varphi\left(\frac{\gamma_{1}+\gamma_{2}}{2}\right) \leq \frac{1}{\gamma_{2}-\gamma_{1}} \int_{0}^{1} \varphi(\tau) \mathrm{d} \tau \leq \frac{\varphi\left(\gamma_{1}\right)+\varphi\left(\gamma_{2}\right)}{2} .
$$

This generalized fractional integral operators had attracted the attention of many scholars. For example, Wu et al. [2] gave three fundamental integral identities via fractional integrals with exponential kernels to establish several Hermite-Hadamard-type inequalities. Zhou et al. [3] derived some parameterized fractional integrals with exponential kernels inequalities for convex mappings. For more information associated with fractional integrals with exponential kernels see reference in [4] [5].

The concept of $m$-convex mappings was introduced by Toader in [6]. It is defined as follows.

Definition 1.2. The mapping $\varphi:\left[0, \gamma_{2}\right] \rightarrow \mathbb{R}, \gamma_{2}>0$ is named m-convex mapping, where $m \in(0,1]$, if for all $k_{1}, k_{2} \in\left[0, \gamma_{2}\right]$ and $\tau \in[0,1]$, we have

$$
\varphi\left(\tau k_{1}+m(1-\tau) k_{2}\right) \leq \tau \varphi\left(k_{1}\right)+m(1-\tau) \varphi\left(k_{2}\right)
$$

Due to the wide applications of $m$-convex mapping, many authors have established various integral inequalities related to $m$-convex mappings. In [7], Dragomir presented some properties and inequalities for $m$-convex mappings. In [8], Jleli et al. extended partial results presented in [7] via generalized fractional integrals. In [9], Farid and Abbas gave some general fractional integral inequalities for $m$-convex mappings associated with generalized Mittag-Leffer mapping. For other works involving $m$-convex mappings, we refer an interseted reader to [10] [11] [12].

These studies motivated us to establish some fractional integrals with exponential kernels inequalities for $m$-convex mappings. We considered two forms of $m$-convex combination to get certain midpoint type and trapezoid type inequalities. We gave new bounds for these inequalities and laid a foundation for their 
application in numerical integration. Some results of this article would provide generalizations of those given in earlier works.

\section{Main Results}

In this part, we mainly establish some fractional integral inequalities based on the properties of $m$-convex functions.

Theorem 2.1. Let $\varphi:[0, \infty) \rightarrow \mathbb{R}$ be a m-convex function with $m \in(0,1]$ and $0 \leq \gamma_{1}<m \gamma_{2}$. If $\varphi \in L^{1}\left[\gamma_{1}, m \gamma_{2}\right]$, then the following inequality exists.

$$
\frac{\mu}{m \gamma_{2}-\gamma_{1}}\left[I_{\gamma_{1}^{+}}^{\mu}\left(m \gamma_{2}\right)+I_{m \gamma_{2}^{-}}^{\mu}\left(\gamma_{1}\right)\right] \leq \frac{1-\mathrm{e}^{-\delta}}{\delta}\left[\varphi\left(\gamma_{1}\right)+m \varphi\left(\gamma_{2}\right)\right] \text {, }
$$

where $\delta=\frac{1-\mu}{\mu}\left(m \gamma_{2}-\gamma_{1}\right)$.

Proof. By means of $m$-convexity of $\varphi$, one has

$$
\varphi\left(\tau \gamma_{1}+m(1-\tau) \gamma_{2}\right) \leq \tau \varphi\left(\gamma_{1}\right)+m(1-\tau) \varphi\left(\gamma_{2}\right)
$$

and

$$
\varphi\left((1-\tau) \gamma_{1}+m \tau \gamma_{2}\right) \leq(1-\tau) \varphi\left(\gamma_{1}\right)+m \tau \varphi\left(\gamma_{2}\right)
$$

Adding the above inequalities, we deduce

$$
\varphi\left(\tau \gamma_{1}+m(1-\tau) \gamma_{2}\right)+\varphi\left((1-\tau) \gamma_{1}+m \tau \gamma_{2}\right) \leq \varphi\left(\gamma_{1}\right)+m \varphi\left(\gamma_{2}\right)
$$

We can obtain the desired inequality by multiplying (2.2) with $\mathrm{e}^{-\theta \tau}$ and then integrating over $[0,1]$ with respect to $\mathrm{d} \tau$. Since

$$
\begin{aligned}
& \int_{0}^{1} \mathrm{e}^{-\delta \tau}\left[\varphi\left(\tau \gamma_{1}+m(1-\tau) \gamma_{2}\right)+\varphi\left((1-\tau) \gamma_{1}+m \tau \gamma_{2}\right)\right] \mathrm{d} \tau \\
& =\int_{0}^{1} \mathrm{e}^{-\delta \tau} \varphi\left(\tau \gamma_{1}+m(1-\tau) \gamma_{2}\right) \mathrm{d} \tau+\int_{0}^{1} \varphi\left((1-\tau) \gamma_{1}+m \tau \gamma_{2}\right) \mathrm{d} \tau \\
& =\frac{1}{m \gamma_{2}-\gamma_{1}} \int_{\gamma_{1}}^{m \gamma_{2}} \mathrm{e}^{-\frac{1-\mu}{\mu}\left(m \gamma_{2}-\gamma_{1}\right) \frac{m \gamma_{2}-x}{m \gamma_{2}-\gamma_{1}}} \varphi(x) \mathrm{d} x \\
& +\frac{1}{m \gamma_{2}-\gamma_{1}} \int_{\gamma_{1}}^{m \gamma_{2}} \mathrm{e}^{-\frac{1-\mu}{\mu}\left(m \gamma_{2}-\gamma_{1}\right) \frac{x-\gamma_{1}}{m \gamma_{2}-\gamma_{1}}} \varphi(x) \mathrm{d} x \\
& =\frac{\mu}{m \gamma_{2}-\gamma_{1}}\left[I_{\gamma_{1}^{+}}^{\mu}\left(m \gamma_{2}\right)+I_{m \gamma_{2}^{-}}^{\mu}\left(\gamma_{1}\right)\right]
\end{aligned}
$$

and

$$
\int_{0}^{1} \mathrm{e}^{-\delta}\left[\varphi\left(\gamma_{1}\right)+m \varphi\left(\gamma_{2}\right)\right] \mathrm{d} \tau=\frac{1-\mathrm{e}^{-\delta}}{\delta}\left[\varphi\left(\gamma_{1}\right)+m \varphi\left(\gamma_{2}\right)\right] .
$$

This ends the proof.

Corollary 2.1. If we consider $m=1$ in Theorem 2.1, then we have right part of inequality (8) in [1].

To obtain trapezoid type inequality related to fractional integrals with exponential kernels, we need the following lemma.

Lemma 2.1. Assuming $\varphi:\left[\gamma_{1}, m \gamma_{2}\right] \rightarrow \mathbb{R}$ is a differentiable mapping with $0 \leq \gamma_{1}<m \gamma_{2}<\infty$ and $0<m \leq 1$. If $\varphi^{\prime} \in L^{1}\left(\left[\gamma_{1}, m \gamma_{2}\right]\right)$, then the following identity holds. 


$$
\begin{aligned}
\Omega\left(\gamma_{1}, \gamma_{2}, \delta, \tau\right)= & \frac{m \gamma_{2}-\gamma_{1}}{2\left(1-\mathrm{e}^{-\delta}\right)}\left[\int_{0}^{1} \mathrm{e}^{-\delta \tau} \varphi^{\prime}\left(\tau \gamma_{1}+m(1-\tau) \gamma_{2}\right) \mathrm{d} \tau\right. \\
& \left.-\int_{0}^{1} \mathrm{e}^{-\delta(1-\tau)} \varphi^{\prime}\left(\tau \gamma_{1}+m(1-\tau) \gamma_{2}\right) \mathrm{d} \tau\right],
\end{aligned}
$$

where $\delta=\frac{1-\mu}{\mu}\left(m \gamma_{2}-\gamma_{1}\right)$ and

$$
\Omega\left(\gamma_{1}, \gamma_{2}, \delta, \tau\right):=\frac{\varphi\left(\gamma_{1}\right)+\varphi\left(m \gamma_{2}\right)}{2}-\frac{1-\mu}{2\left(1-\mathrm{e}^{-\delta}\right)}\left[I_{\gamma_{1}^{+}}^{\mu} \varphi\left(m \gamma_{2}\right)+I_{m \gamma_{\overline{2}}^{-}}^{\mu} \varphi\left(\gamma_{1}\right)\right] .
$$

Proof. Integrating the following formula by parts, we have

$$
\begin{aligned}
& \int_{0}^{1} \mathrm{e}^{-\delta \tau} \varphi^{\prime}\left(\tau \gamma_{1}+m(1-\tau) \gamma_{2}\right) \mathrm{d} \tau-\int_{0}^{1} \mathrm{e}^{-\delta(1-\tau)} \varphi^{\prime}\left(\tau \gamma_{1}+m(1-\tau) \gamma_{2}\right) \mathrm{d} \tau \\
&=\frac{1}{\gamma_{1}-m \gamma_{2}} \int_{0}^{1} \mathrm{e}^{-\delta \tau} \mathrm{d}\left(\varphi\left(\tau \gamma_{1}+m(1-\tau) \gamma_{2}\right)\right) \\
&-\frac{1}{\gamma_{1}-m \gamma_{2}} \int_{0}^{1} \mathrm{e}^{-\delta(1-\tau)} \mathrm{d}\left(\varphi\left(\tau \gamma_{1}+m(1-\tau) \gamma_{2}\right)\right) \\
&= \frac{1}{\gamma_{1}-m \gamma_{2}}\left[\left.\mathrm{e}^{-\delta \tau} \varphi\left(\tau \gamma_{1}+m(1-\tau) \gamma_{2}\right)\right|_{0} ^{1}-\int_{0}^{1} \varphi\left(\tau \gamma_{1}+m(1-\tau) \gamma_{2}\right) \mathrm{d}\left(\mathrm{e}^{-\delta \tau}\right)\right] \\
&-\frac{1}{\gamma_{1}-m \gamma_{2}}\left[\left.\mathrm{e}^{-\delta(1-\tau)} \varphi\left(\tau \gamma_{1}+m(1-\tau) \gamma_{2}\right)\right|_{0} ^{1}\right. \\
&\left.-\int_{0}^{1} \varphi\left(\tau \gamma_{1}+m(1-\tau) \gamma_{2}\right) \mathrm{d}\left(\mathrm{e}^{-\delta(1-\tau)}\right)\right] \\
&= \frac{1}{m \gamma_{2}-\gamma_{1}}\left[\left(1-\mathrm{e}^{-\delta}\right)\left(\varphi\left(\gamma_{1}\right)+\varphi\left(m \gamma_{2}\right)\right)-\delta \int_{0}^{1} \mathrm{e}^{-\delta \tau} \varphi\left(\tau \gamma_{1}+m(1-\tau) \gamma_{2}\right) \mathrm{d} \tau\right. \\
&\left.-\delta \int_{0}^{1} \mathrm{e}^{-\delta(1-\tau)} \varphi\left(\tau \gamma_{1}+m(1-\tau) \gamma_{2}\right) \mathrm{d} \tau\right] \\
&= \frac{1}{m \gamma_{2}-\gamma_{1}}\left(\left(1-\mathrm{e}^{-\delta}\right)\left(\varphi\left(\gamma_{1}\right)+\varphi\left(m \gamma_{2}\right)\right)-(1-\mu)\left[I_{\gamma_{1}^{+}}^{\mu} \varphi\left(m \gamma_{2}\right)+I_{m \gamma_{2}}^{\mu} \varphi\left(\gamma_{1}\right)\right]\right) .
\end{aligned}
$$

Multiplying both sides of (2.5) by $\frac{m \gamma_{2}-\gamma_{1}}{2\left(1-\mathrm{e}^{-\delta}\right)}$, we have the conclusion (2.3). The proof is completed.

Theorem 2.2. Let $\varphi$ be defined as in Lemma 2.1. If $\varphi^{\prime}$ is m-convex on $\left[\gamma_{1}, m \gamma_{2}\right]$ for some fixed $m \in[0,1]$, then the following inequality for fractional integrals with exponential kernels holds.

$$
\left|\Omega\left(\gamma_{1}, \gamma_{2}, \delta, \tau\right)\right| \leq \frac{m \gamma_{2}-\gamma_{1}}{2 \delta} \tanh \left(\frac{\delta}{4}\right)\left[\left|\varphi^{\prime}\left(\gamma_{1}\right)\right|+m\left|\varphi^{\prime}\left(\gamma_{2}\right)\right|\right] .
$$

Proof. Applying Lemma 2.1 and convexity of $\left|\varphi^{\prime}\right|$, we obtain

$$
\begin{aligned}
& \left|\Omega\left(\gamma_{1}, \gamma_{2}, \delta, \tau\right)\right| \\
& \leq \frac{m \gamma_{2}-\gamma_{1}}{2\left(1-\mathrm{e}^{-\delta}\right)}\left[\int_{0}^{1}\left|\mathrm{e}^{-\delta \tau} \varphi^{\prime}\left(\tau \gamma_{1}+m(1-\tau) \gamma_{2}\right)-\mathrm{e}^{-\delta(1-\tau)} \varphi^{\prime}\left(\tau \gamma_{1}+m(1-\tau) \gamma_{2}\right)\right| \mathrm{d} \tau\right] \\
& \leq \frac{m \gamma_{2}-\gamma_{1}}{2\left(1-\mathrm{e}^{-\delta}\right)}\left[\int_{0}^{1}\left|\mathrm{e}^{-\delta \tau}-\mathrm{e}^{-\delta(1-\tau)}\right|\left|\varphi^{\prime}\left(\tau \gamma_{1}+m(1-\tau) \gamma_{2}\right)\right| \mathrm{d} \tau\right] \\
& \leq \frac{m \gamma_{2}-\gamma_{1}}{2\left(1-\mathrm{e}^{-\delta}\right)}\left[\int_{0}^{1}\left|\mathrm{e}^{-\delta \tau}-\mathrm{e}^{-\delta(1-\tau)}\right|\left(\tau \varphi^{\prime}\left(\gamma_{1}\right)+m(1-\tau) \varphi^{\prime}\left(\gamma_{2}\right)\right) \mathrm{d} \tau\right] .
\end{aligned}
$$


By calculation, we have

$$
\int_{0}^{1} t\left|\mathrm{e}^{-\delta \tau}-\mathrm{e}^{-\delta(1-\tau)}\right| \varphi^{\prime}\left(\gamma_{1}\right) \mathrm{d} \tau=\frac{1-2 \mathrm{e}^{-\frac{\delta}{2}}+\mathrm{e}^{-\delta}}{\delta} \varphi^{\prime}\left(\gamma_{1}\right)
$$

and

$$
\int_{0}^{1} m(1-t)\left|\mathrm{e}^{-\delta \tau}-\mathrm{e}^{-\delta(1-\tau)}\right| \varphi^{\prime}\left(\gamma_{2}\right) \mathrm{d} \tau=m \frac{1-2 \mathrm{e}^{-\frac{\delta}{2}}+\mathrm{e}^{-\delta}}{\delta} \varphi^{\prime}\left(\gamma_{2}\right) .
$$

Utilizing inequality (2.8) and inequality (2.9) in inequality (2.7), we have

$$
\left|\Omega\left(\gamma_{1}, \gamma_{2}, \delta, \tau\right)\right| \leq \frac{m \gamma_{2}-\gamma_{1}}{2 \delta} \tanh \left(\frac{\delta}{4}\right)\left[\left|\varphi^{\prime}\left(\gamma_{1}\right)\right|+m\left|\varphi^{\prime}\left(\gamma_{2}\right)\right|\right] .
$$

The proof is completed.

Corollary 2.2. If we consider $m=1$ in Theorem 2.2, then we can deduce Theorem 3 in [1].

Theorem 2.3. Let $\varphi:[0, \infty) \rightarrow \mathbb{R}$ be a m-convex function with $m \in(0,1]$ and $0 \leq \gamma_{1}<\gamma_{2}$. If $\varphi \in L^{1}\left[\gamma_{1}, \gamma_{2}\right]$, then the following inequality exists.

$$
\begin{aligned}
& \frac{\mu}{\gamma_{2}-\gamma_{1}}\left[I_{\gamma_{2}^{-}}^{\mu} \varphi\left(\gamma_{1}\right)+I_{\gamma_{1}^{+}}^{\mu} \varphi\left(\gamma_{+}\right)\right] \\
& \leq \frac{-\rho \mathrm{e}^{-\rho}-\mathrm{e}^{\rho}+1}{\rho^{2}}\left[\varphi\left(\gamma_{1}\right)+\varphi\left(\gamma_{2}\right)\right]+m \frac{\mathrm{e}^{-\rho}+\rho-1}{\rho^{2}}\left[\varphi\left(\frac{\gamma_{1}}{m}\right)+\varphi\left(\frac{\gamma_{2}}{m}\right)\right],
\end{aligned}
$$

where $\rho=\frac{1-\mu}{\mu}\left(\gamma_{2}-\gamma_{1}\right)$.

Proof. By means of $m$-convexity of $\varphi$, we deduce

$$
\varphi\left(\tau \gamma_{1}+(1-\tau) \gamma_{2}\right) \leq \tau \varphi\left(\gamma_{1}\right)+m(1-\tau) \varphi\left(\frac{\gamma_{2}}{m}\right)
$$

and

$$
\varphi\left(\tau \gamma_{2}+(1-\tau) \gamma_{1}\right) \leq \tau \varphi\left(\gamma_{2}\right)+m(1-\tau) \varphi\left(\frac{\gamma_{1}}{m}\right)
$$

Multiplying above-mentioned inequalities with $\mathrm{e}^{-\rho \tau}$ and then integrating over $[0,1]$ with respect to $\mathrm{d} \tau$, we get

$$
\begin{aligned}
& \int_{0}^{1} \mathrm{e}^{-\rho \tau} \varphi\left(\tau \gamma_{1}+(1-\tau) \gamma_{2}\right) \mathrm{d} \tau \\
& =\frac{1}{\gamma_{2}-\gamma_{1}} \int_{\gamma_{1}}^{\gamma_{2}} \mathrm{e}^{-\frac{1-\mu}{\mu}\left(\gamma_{2}-x\right)} \varphi(x) \mathrm{d} x=\frac{\mu}{\gamma_{2}-\gamma_{1}} I_{\gamma_{2}^{-}}^{\mu} \varphi\left(\gamma_{1}\right) \\
& \leq \int_{0}^{1} \mathrm{e}^{-\rho \tau}\left[\tau \varphi\left(\gamma_{1}\right)+m(1-\tau) \varphi\left(\frac{\gamma_{2}}{m}\right)\right] \mathrm{d} \tau \\
& =\frac{-\rho \mathrm{e}^{-\rho}-\mathrm{e}^{\rho}+1}{\rho^{2}} \varphi\left(\gamma_{1}\right)+m \frac{\mathrm{e}^{-\rho}+\rho-1}{\rho^{2}} \varphi\left(\frac{\gamma_{2}}{m}\right)
\end{aligned}
$$

and 


$$
\begin{aligned}
& \int_{0}^{1} \mathrm{e}^{-\rho \tau} \varphi\left(\tau \gamma_{2}+(1-\tau) \gamma_{1}\right) \mathrm{d} \tau \\
& =\frac{1}{\gamma_{2}-\gamma_{1}} \int_{\gamma_{1}}^{\gamma_{2}} \mathrm{e}^{-\frac{1-\mu}{\mu}\left(x-\gamma_{1}\right)} \varphi(x) \mathrm{d} x=\frac{\mu}{\gamma_{2}-\gamma_{1}} I_{\gamma_{1}^{+}}^{\mu} \varphi\left(\gamma_{2}\right) \\
& \leq \int_{0}^{1} \mathrm{e}^{-\rho \tau}\left[\tau \varphi\left(\gamma_{2}\right)+m(1-\tau) \varphi\left(\frac{\gamma_{1}}{m}\right)\right] \mathrm{d} \tau \\
& =\frac{-\rho \mathrm{e}^{-\rho}-\mathrm{e}^{\rho}+1}{\rho^{2}} \varphi\left(\gamma_{2}\right)+m \frac{\mathrm{e}^{-\rho}+\rho-1}{\rho^{2}} \varphi\left(\frac{\gamma_{1}}{m}\right) .
\end{aligned}
$$

By adding (2.11) and (2.12) together, we have completed the proof.

Corollary 2.3. If we consider $\mu \rightarrow 1$ i.e. $\rho=\frac{1-\mu}{\mu}\left(\gamma_{2}-\gamma_{1}\right) \rightarrow 0$ in Theorem 2.3 , observe that

$$
\lim _{\rho \rightarrow 0} \frac{-\rho \mathrm{e}^{-\rho}-\mathrm{e}^{\rho}+1}{\rho^{2}}=1
$$

and

$$
\lim _{\rho \rightarrow 0} \frac{\mathrm{e}^{-\rho}+\rho-1}{\rho^{2}}=\frac{1}{2}
$$

then we have

$$
\frac{2}{\gamma_{2}-\gamma_{1}} \int_{\gamma_{1}}^{\gamma_{2}} \varphi(\tau) \mathrm{d} \tau \leq\left[\varphi\left(\gamma_{1}\right)+\varphi\left(\gamma_{2}\right)\right]+\frac{m}{2}\left[\varphi\left(\frac{\gamma_{1}}{m}\right)+\varphi\left(\frac{\gamma_{2}}{m}\right)\right] .
$$

Theorem 2.4. Under the assumptions of Theorem 2.3, if we take

$$
\begin{aligned}
\theta & =\frac{\mu}{1-\mu} \frac{\gamma_{2}-\gamma_{1}}{2}, \text { then the resulting expression holds. } \\
& \frac{\mu}{\gamma_{2}-\gamma_{1}}\left[I_{\gamma_{1}^{+}}^{\mu} \varphi\left(\frac{\gamma_{1}+\gamma_{2}}{2}\right)+I_{\gamma_{2}^{-}}^{\mu} \varphi\left(\frac{\gamma_{1}+\gamma_{2}}{2}\right)\right] \\
& \leq \frac{-2 \theta \mathrm{e}^{-\theta}-\mathrm{e}^{-\theta}+\theta+1}{4 \theta^{2}}\left[\varphi\left(\gamma_{1}\right)+\varphi\left(\gamma_{2}\right)\right]+m \frac{\mathrm{e}^{-\theta}+\theta-1}{4 \theta^{2}}\left[\varphi\left(\frac{\gamma_{1}}{m}\right)+\varphi\left(\frac{\gamma_{2}}{m}\right)\right] .
\end{aligned}
$$

Proof. Since $\varphi$ is $m$-convex, we have

$$
\varphi\left(\frac{1+\tau}{2} \gamma_{1}+\frac{1-\tau}{2} \gamma_{2}\right) \leq \frac{1+\tau}{2} \varphi\left(\gamma_{1}\right)+m \frac{1-\tau}{2} \varphi\left(\frac{\gamma_{2}}{m}\right)
$$

and

$$
\varphi\left(\frac{1+\tau}{2} \gamma_{2}+\frac{1-\tau}{2} \gamma_{1}\right) \leq \frac{1+\tau}{2} \varphi\left(\gamma_{2}\right)+m \frac{1-\tau}{2} \varphi\left(\frac{\gamma_{1}}{m}\right) .
$$

Adding inequality (2.15) and inequality (2.16) together and then multiplying by $\mathrm{e}^{-\theta \tau}$, we get

$$
\begin{aligned}
& \mathrm{e}^{-\theta \tau}\left[\varphi\left(\frac{1+\tau}{2} \gamma_{2}+\frac{1-\tau}{2} \gamma_{1}\right)+\varphi\left(\frac{1+\tau}{2} \gamma_{1}+\frac{1-\tau}{2} \gamma_{2}\right)\right] \\
& \leq \mathrm{e}^{-\theta \tau}\left(\frac{1+\tau}{2}\left[\varphi\left(\gamma_{1}\right)+\varphi\left(\gamma_{2}\right)\right]+m \frac{1-\tau}{2}\left[\varphi\left(\frac{\gamma_{1}}{m}\right)+\varphi\left(\frac{\gamma_{2}}{m}\right)\right]\right) .
\end{aligned}
$$


Integrating on both sides of inequality (2.17) respect to $\tau$ over $[0,1]$, we have completed the proof. Since

$$
\begin{aligned}
& \int_{0}^{1} \mathrm{e}^{-\theta \tau}\left[\varphi\left(\frac{1+\tau}{2} \gamma_{2}+\frac{1-\tau}{2} \gamma_{1}\right)+\varphi\left(\frac{1+\tau}{2} \gamma_{1}+\frac{1-\tau}{2} \gamma_{2}\right)\right] \mathrm{d} \tau \\
& =\int_{0}^{1} \mathrm{e}^{-\theta \tau} \varphi\left(\frac{1+\tau}{2} \gamma_{2}+\frac{1-\tau}{2} \gamma_{1}\right) \mathrm{d} \tau+\int_{0}^{1} \mathrm{e}^{-\theta \tau} \varphi\left(\frac{1+\tau}{2} \gamma_{1}+\frac{1-\tau}{2} \gamma_{2}\right) \mathrm{d} \tau \\
& =\frac{2}{\gamma_{2}-\gamma_{1}} \int_{\gamma_{1}}^{\frac{\gamma_{1}+\gamma_{2}}{2}} \mathrm{e}^{-\frac{1-\mu \gamma_{2}-\gamma_{1}\left(\gamma_{1}+\gamma_{1}\right)-2 x}{\gamma_{2}-\gamma_{1}}} \varphi(x) \mathrm{d} x \\
& \quad+\frac{2}{\gamma_{2}-\gamma_{1}} \int_{\frac{\gamma_{2}+\gamma_{1}}{2}}^{\gamma_{2}} \mathrm{e}^{-\frac{1-\mu \gamma_{2}-\gamma_{1}}{2} \frac{2 x-\left(\gamma_{1}+\gamma_{1}\right)}{\gamma_{2}-\gamma_{1}}} \varphi(x) \mathrm{d} x \\
& =\frac{2 \mu}{\gamma_{2}-\gamma_{1}}\left[I_{\gamma_{1}^{+}}^{\mu} \varphi\left(\frac{\gamma_{1}+\gamma_{2}}{2}\right)+I_{\gamma_{2}}^{\mu} \varphi\left(\frac{\gamma_{1}+\gamma_{2}}{2}\right)\right]
\end{aligned}
$$

and

$$
\begin{aligned}
& \int_{0}^{1} \mathrm{e}^{-\theta \tau}\left(\frac{1+\tau}{2}\left[\varphi\left(\gamma_{1}\right)+\varphi\left(\gamma_{2}\right)\right]+m \frac{1-\tau}{2}\left[\varphi\left(\frac{\gamma_{1}}{m}\right)+\varphi\left(\frac{\gamma_{2}}{m}\right)\right]\right) \mathrm{d} \tau \\
& =\frac{-2 \theta \mathrm{e}^{-\theta}-\mathrm{e}^{-\theta}+\theta+1}{2 \theta^{2}}\left(\varphi\left(\gamma_{1}\right)+\varphi\left(\gamma_{2}\right)\right)+m \frac{\mathrm{e}^{-\theta}+\theta-1}{2 \theta^{2}}\left(\varphi\left(\frac{\gamma_{1}}{m}\right)+\varphi\left(\frac{\gamma_{2}}{m}\right)\right) .
\end{aligned}
$$

We now use the following two lemmas, which are presented in [13], to obtain some mid-point type and trapezoid type inequalities.

Lemma 2.2. Assuming $\varphi:\left[\gamma_{1}, \gamma_{2}\right] \rightarrow \mathbb{R}$ is a differentiable mapping, such that $\varphi \in L^{1}\left(\left[\gamma_{1}, \gamma_{2}\right]\right)$ with $0 \leq \gamma_{1}<\gamma_{2}<\infty$, then the following identity holds.

$$
\begin{aligned}
& -\frac{1-\mu}{2\left(1-\mathrm{e}^{-\theta}\right)}\left[I_{\frac{\gamma_{2}+\gamma_{1}}{2}}^{\mu} \varphi\left(\gamma_{1}\right)+I_{\frac{\gamma_{2}+\gamma_{1}}{2}}^{\mu} \varphi\left(\gamma_{2}\right)\right]+\varphi\left(\frac{\gamma_{1}+\gamma_{2}}{2}\right) \\
& =\frac{\gamma_{2}-\gamma_{1}}{4\left(1-\mathrm{e}^{-\theta}\right)}\left\{\int_{0}^{1}\left[\mathrm{e}^{-\theta \tau}-1\right] \varphi^{\prime}\left(\frac{\tau}{2} \gamma_{1}+\frac{2-\tau}{2} \gamma_{2}\right) \mathrm{d} \tau\right. \\
& \left.-\int_{0}^{1}\left[\mathrm{e}^{-\theta \tau}-1\right] \varphi^{\prime}\left(\frac{2-\tau}{2} \gamma_{1}+\frac{\tau}{2} \gamma_{2}\right) \mathrm{d} \tau\right\} .
\end{aligned}
$$

Lemma 2.3. Assuming $\varphi:\left[\gamma_{1}, \gamma_{2}\right] \rightarrow \mathbb{R}$ is a positive convex mapping, such that $\varphi \in L^{1}\left(\left[\gamma_{1}, \gamma_{2}\right]\right)$ with $0 \leq \gamma_{1}<\gamma_{2}<\infty$, then the following identity exists.

$$
\begin{aligned}
& \frac{\varphi\left(\gamma_{1}+\gamma_{2}\right)}{2}-\frac{1-\mu}{2\left(1-\mathrm{e}^{-\theta}\right)}\left[I_{\gamma_{2}}^{\mu} \varphi\left(\frac{\gamma_{1}+\gamma_{2}}{2}\right)+I_{\gamma_{1}^{+}}^{\mu} \varphi\left(\frac{\gamma_{1}+\gamma_{2}}{2}\right)\right] \\
& =\frac{\gamma_{2}-\gamma_{1}}{4\left(\mathrm{e}^{-\theta}-1\right)}\left\{\int_{0}^{1}\left[\mathrm{e}^{-\theta \tau}-1\right] \varphi^{\prime}\left(\frac{1-\tau}{2} \gamma_{1}+\frac{1+\tau}{2} \gamma_{2}\right) \mathrm{d} \tau\right. \\
& \left.\quad-\int_{0}^{1}\left[\mathrm{e}^{-\theta \tau}-1\right] \varphi^{\prime}\left(\frac{1+\tau}{2} \gamma_{1}+\frac{1-\tau}{2} \gamma_{2}\right) \mathrm{d} \tau\right\} .
\end{aligned}
$$

Theorem 2.5. Under the assumptions of lemma 2.2, if $\left|\varphi^{\prime}\right|$ is m-convex on $\left[\gamma_{1}, \gamma_{2}\right]$, then the resulting expression holds. 


$$
\begin{aligned}
& \left|-\frac{1-\mu}{2\left(1-\mathrm{e}^{-\theta}\right)}\left[I_{\frac{\gamma_{2}+\gamma_{1}}{2}}^{\mu} \varphi\left(\gamma_{1}\right)+I_{\frac{\gamma_{2}+\gamma_{1}}{2}}^{\mu} \varphi\left(\gamma_{2}\right)\right]+\varphi\left(\frac{\gamma_{1}+\gamma_{2}}{2}\right)\right| \\
& \leq \frac{\gamma_{2}-\gamma_{1}}{4 \theta\left(1-\mathrm{e}^{-\theta}\right)}\left(\mathrm{e}^{-\theta}-1+\theta\right)\left[\left|\varphi^{\prime}\left(\gamma_{1}\right)\right|+m\left|\varphi^{\prime}\left(\frac{\gamma_{2}}{m}\right)\right|\right] .
\end{aligned}
$$

Proof. Applying Lemma 2.2 and the convexity of $\left|\varphi^{\prime}\right|$, one has

$$
\begin{aligned}
& \left|-\frac{1-\mu}{2\left(1-\mathrm{e}^{-\theta}\right)}\left[I_{\frac{\gamma_{2}+\gamma_{1}}{2}}^{\mu} \varphi\left(\gamma_{1}\right)+I_{\frac{\gamma_{2}+\gamma_{1}}{2}}^{\mu} \varphi\left(\gamma_{2}\right)\right]+\varphi\left(\frac{\gamma_{1}+\gamma_{2}}{2}\right)\right| \\
& \leq \frac{\gamma_{2}-\gamma_{1}}{4\left(1-\mathrm{e}^{-\theta}\right)}\left[\int_{0}^{1} \mathrm{e}^{-\theta \tau}-1|| \varphi^{\prime}\left(\frac{\tau}{2} \gamma_{1}+m \frac{2-\tau}{2} \frac{\gamma_{2}}{m}\right) \mid \mathrm{d} \tau\right. \\
& \left.\quad+\int_{0}^{1}\left|\mathrm{e}^{-\theta \tau}-1\right|\left|\varphi^{\prime}\left(\frac{2-\tau}{2} \gamma_{1}+m \frac{\tau}{2} \frac{\gamma_{2}}{m}\right)\right| \mathrm{d} \tau\right] \\
& \leq \frac{\gamma_{2}-\gamma_{1}}{4\left(1-\mathrm{e}^{-\theta}\right)}\left[\int_{0}^{1}\left|\mathrm{e}^{-\theta \tau}-1\right|\left(\frac{\tau}{2}\left|\varphi^{\prime}\left(\gamma_{1}\right)\right|+m \frac{2-\tau}{2}\left|\varphi^{\prime}\left(\frac{\gamma_{2}}{m}\right)\right|\right) \mathrm{d} \tau\right. \\
& \left.\quad+\int_{0}^{1}\left|\mathrm{e}^{-\theta \tau}-1\right|\left(\frac{2-\tau}{2}\left|\varphi^{\prime}\left(\gamma_{1}\right)\right|+m \frac{\tau}{2}\left|\varphi^{\prime}\left(\frac{\gamma_{2}}{m}\right)\right|\right) \mathrm{d} \tau\right],
\end{aligned}
$$

where we use the fact that

$$
\int_{0}^{1} \frac{\tau}{2}\left|\mathrm{e}^{-\theta \tau}-1\right| \mathrm{d} \tau=\frac{1}{4}-\frac{-\theta \mathrm{e}^{-\theta}-\mathrm{e}^{-\theta}+1}{2 \theta^{2}}
$$

and

$$
\int_{0}^{1} \frac{2-\tau}{2}\left|\mathrm{e}^{-\theta \tau}-1\right| \mathrm{d} \tau=\frac{-\theta \mathrm{e}^{-\theta}-\mathrm{e}^{-\theta}+1}{2 \theta^{2}}+\frac{\mathrm{e}^{-\theta}-1}{\theta}+\frac{3}{4} .
$$

After suitable arrangements, we obtain

$$
\begin{aligned}
& \left|-\frac{1-\mu}{2\left(1-\mathrm{e}^{-\theta}\right)}\left[I_{\frac{\gamma_{2}+\gamma_{1}}{2}}^{\mu} \varphi\left(\gamma_{1}\right)+I_{\frac{\gamma_{2}+\gamma_{1}}{2}}^{\mu} \varphi\left(\gamma_{2}\right)\right]+\varphi\left(\frac{\gamma_{1}+\gamma_{2}}{2}\right)\right| \\
& \leq \frac{\gamma_{2}-\gamma_{1}}{4 \theta\left(1-\mathrm{e}^{-\theta}\right)}\left(\mathrm{e}^{-\theta}-1+\theta\right)\left[\left|\varphi^{\prime}\left(\gamma_{1}\right)\right|+m\left|\varphi^{\prime}\left(\frac{\gamma_{2}}{m}\right)\right|\right] .
\end{aligned}
$$

This ends the proof.

Theorem 2.6. Under the assumptions of Lemma 2.3, if $\left|\varphi^{\prime}\right|$ is m-convex on $\left[\gamma_{1}, \gamma_{2}\right]$, then the resulting expression holds.

$$
\begin{aligned}
& \left|\frac{\varphi\left(\gamma_{1}+\gamma_{2}\right)}{2}-\frac{1-\mu}{2\left(1-\mathrm{e}^{-\theta}\right)}\left[I_{\gamma_{2}^{-}}^{\mu} \varphi\left(\frac{\gamma_{1}+\gamma_{2}}{2}\right)+I_{\gamma_{1}^{+}}^{\mu} \varphi\left(\frac{\gamma_{1}+\gamma_{2}}{2}\right)\right]\right| \\
& \leq \frac{\gamma_{2}-\gamma_{1}}{4 \theta\left(1-\mathrm{e}^{-\theta}\right)}\left(\mathrm{e}^{-\theta}-1+\theta\right)\left[\left|\varphi^{\prime}\left(\gamma_{1}\right)\right|+m\left|\varphi^{\prime}\left(\frac{\gamma_{2}}{m}\right)\right|\right] .
\end{aligned}
$$

Proof. Applying Lemma 2.3 and the convexity of $\left|\varphi^{\prime}\right|$, one has 


$$
\begin{aligned}
& \left|\frac{\varphi\left(\gamma_{1}+\gamma_{2}\right)}{2}-\frac{1-\mu}{2\left(1-\mathrm{e}^{-\theta}\right)}\left[I_{\gamma_{2}^{-}}^{\mu} \varphi\left(\frac{\gamma_{1}+\gamma_{2}}{2}\right)+I_{\gamma_{1}^{+}}^{\mu} \varphi\left(\frac{\gamma_{1}+\gamma_{2}}{2}\right)\right]\right| \\
& \leq \frac{\gamma_{2}-\gamma_{1}}{4\left(1-\mathrm{e}^{-\theta}\right)}\left[\int_{0}^{1}\left|\mathrm{e}^{-\theta \tau}-1\right|\left|\varphi^{\prime}\left(\frac{1-\tau}{2} \gamma_{1}+m \frac{1+\tau}{2} \frac{\gamma_{2}}{m}\right)\right| \mathrm{d} \tau\right. \\
& \left.\quad+\int_{0}^{1}\left|\mathrm{e}^{-\theta \tau}-1\right|\left|\varphi^{\prime}\left(\frac{1+\tau}{2} \gamma_{1}+m \frac{1-\tau}{2} \frac{\gamma_{2}}{m}\right)\right| \mathrm{d} \tau\right] \\
& \leq \frac{\gamma_{2}-\gamma_{1}}{4\left(1-\mathrm{e}^{-\theta}\right)}\left[\int_{0}^{1}\left|\mathrm{e}^{-\theta \tau}-1\right|\left(\frac{1-\tau}{2}\left|\varphi^{\prime}\left(\gamma_{1}\right)\right|+m \frac{1+\tau}{2}\left|\varphi^{\prime}\left(\frac{\gamma_{2}}{m}\right)\right|\right) \mathrm{d} \tau\right. \\
& \left.\quad+\int_{0}^{1}\left|\mathrm{e}^{-\theta \tau}-1\right|\left(\frac{1+\tau}{2}\left|\varphi^{\prime}\left(\gamma_{1}\right)\right|+m \frac{1-\tau}{2}\left|\varphi^{\prime}\left(\frac{\gamma_{2}}{m}\right)\right|\right) \mathrm{d} \tau\right],
\end{aligned}
$$

where we use the fact that

$$
\int_{0}^{1} \frac{1-\tau}{2}\left|\mathrm{e}^{-\theta \tau}-1\right| \mathrm{d} \tau=\frac{1}{4}+\frac{\mathrm{e}^{-\theta}-1}{\theta}+\frac{-\theta \mathrm{e}^{-\theta}-\mathrm{e}^{-\theta}+1}{\theta^{2}}
$$

and

$$
\int_{0}^{1} \frac{1+\tau}{2}\left|\mathrm{e}^{-\theta \tau}-1\right| \mathrm{d} \tau=\frac{3}{4}+\frac{\mathrm{e}^{-\theta}-1}{\theta}-\frac{-\theta \mathrm{e}^{-\theta}-\mathrm{e}^{-\theta}+1}{2 \theta^{2}} .
$$

After suitable arrangements, we obtain

$$
\begin{aligned}
& \left|\frac{\varphi\left(\gamma_{1}+\gamma_{2}\right)}{2}-\frac{1-\mu}{2\left(1-\mathrm{e}^{-\theta}\right)}\left[I_{\gamma_{2}^{2}}^{\mu} \varphi\left(\frac{\gamma_{1}+\gamma_{2}}{2}\right)+I_{\gamma_{1}^{+}}^{\mu} \varphi\left(\frac{\gamma_{1}+\gamma_{2}}{2}\right)\right]\right| \\
& \leq \frac{\gamma_{2}-\gamma_{1}}{4 \theta\left(1-\mathrm{e}^{-\theta}\right)}\left(\mathrm{e}^{-\theta}-1+\theta\right)\left[\left|\varphi^{\prime}\left(\gamma_{1}\right)\right|+m\left|\varphi^{\prime}\left(\frac{\gamma_{2}}{m}\right)\right|\right] .
\end{aligned}
$$

This ends the proof.

\section{Conclusion}

In this article, taking different exponential kernels parameters, we established three fractional integrals inequalities for $m$-convex mappings. Furthermore, we constructed a new lemma to obtain Dragomir-Agarwal inequality for $m$-convex mappings. We emphasized that certain results proved in this article generalize and extend parts of the results provided by Ahmad et al. in [1]. Finally, we gave mid-point type and trapezoid type inequalities for $m$-convex mappings.

\section{Funding}

This work is supported by the General project of Education Department of Hunan Province (No. 19C0359) and General project of Education Department of Hunan Province (No. 19C0377).

\section{Conflicts of Interest}

The authors declare no conflicts of interest regarding the publication of this paper. 


\section{References}

[1] Ahmad, B., Alsaedi, A., Kirane, M. and Torebek, B.T. (2019) Hermite-Hadamard, Hermite-Hadamard-Fejér, Dragomir-Agarwal and Pachpatte Type Inequalities for Convex Functions via New Fractional Integrals. Journal of Computational and Applied Mathematics, 353, 120-129. https://doi.org/10.1016/j.cam.2018.12.030

[2] Wu, X., Wang, J.R. and Zhang, J. (2019) Hermite-Hadamard-Type Inequalities for Convex Functions via the Fractional Integrals with Exponential Kernel. Mathematics, 7, 845. https://doi.org/10.3390/math7090845

[3] Zhou, T.C., Yuan, Z.R., Yang, H.Y. and Du, T.S. (2020) Some Parameterized Inequalities by Means of Fractional Integrals with Exponential Kernels and Their Applications. Journal of Inequalities and Applications, 2020, Article No. 163. https://doi.org/10.1186/s13660-020-02430-9

[4] Dragomir, S.S. and Berikbol, T.T. (2019) Some Hermite-Hademard Type Inequalities in the Class of Hyperbolic p-Convex Functions. Revista de la Real Academia de Ciencias Exactas, Físicas y Naturales. Serie A. Matemáticas, 113, 3413-3423. https://doi.org/10.1007/s13398-019-00708-2

[5] Usat, F., Budak, H., Sarikaya, M.Z. and Yildirm, H. (2017) Some Hermite-Hadamard and Ostrowski Type Inequalities Type Inequalities for Fractional Integral Operators with Exponential Kennel. Acta et Commentationes Universitatis Tartuensis de Mathematica, 23, 1-8. https://doi.org/10.12697/ACUTM.2019.23.03

[6] Toader, G.H. (1984) Some Generalisations of the Convexity. In: Proceedings of Colloquium on Approximation and Optimization, Romania, 329-338.

[7] Dragomir, S.S. (2002) On Some New Inequalities of Hermite-Hadamard Type for $m$-Convex Functions. Tamkang Journal of Mathematics, 33, 45-56. https://doi.org/10.5556/j.tkjm.33.2002.304

[8] Jleli, M., O’Regan, D. and Samet, B. (2017) Some Fractional Integral Inequalities Involving $m$-Convex Functions. Aequationes Mathematicae, 91, 479-490. https://doi.org/10.1007/s00010-017-0470-2

[9] Farid, G. and Abbas, G. (2018) Generalizations of Some Fractional Integral Inequalities for $m$-Convex Functions via Generalized Mittag-Leffler Function. Studia Universitatis Babeş-Bolyai Mathematica, 63, 23-35. https://doi.org/10.24193/subbmath.2018.1.02

[10] Du, T.S., Wang, H., Khan, M.A. and Zhang, Y. (2019) Certain Integral Inequalities Considering Generalized $m$-Convexity on Fractal Sets and Their Applications. Fractals, 27, Article ID: 1950117. https://doi.org/10.1142/S0218348X19501172

[11] Matkowski, J. and Wróbel, M. (2017) Sandwich Theorem for $m$-Convex Functions. Journal of Mathematical Analysis and Applications, 451, 924-930. https://doi.org/10.1016/j.jmaa.2017.02.041

[12] Pavić, Z. and Ardiç, M.A. (2017) The Most Important Inequalities of $m$-Convex Functions. Turkish Journal of Mathematics, 41, 625-635. https://doi.org/10.3906/mat-1604-45

[13] Wang, H. (2021) Certain Fractional Integrals with Exponential Kernels Inequalities Related to Hermite-Hadamard Type. (With Submitted) 